\title{
99mTc-Nanocolloid SPECT/MRI Fusion for the Selective Assessment of Nonenlarged Sentinel Lymph Nodes in Patients with Early-Stage Cervical Cancer
}

\author{
Jacob P. Hoogendam ${ }^{1}$, Ronald P. Zweemer ${ }^{1}$, Monique G.G. Hobbelink ${ }^{2}$, Maurice A.A.J. van den Bosch ${ }^{2}$, \\ René H.M. Verheijen ${ }^{1}$, and Wouter B. Veldhuis ${ }^{2}$ \\ ${ }^{1}$ Department of Gynaecological Oncology, UMC Utrecht Cancer Center, Utrecht, The Netherlands; and ${ }^{2}$ Department of Radiology \\ and Nuclear Medicine, Division Image, University Medical Center Utrecht, Utrecht, The Netherlands
}

\begin{abstract}
We aimed to explore the accuracy of ${ }^{99 m T c ~ S P E C T / M R I ~ f u s i o n ~ f o r ~ t h e ~}$ selective assessment of nonenlarged sentinel lymph nodes (SLNs) for diagnosing metastases in early-stage cervical cancer patients. Methods: We consecutively included stage IA1-IIB1 cervical cancer patients who presented to our tertiary referral center between March 2011 and February 2015. Patients with enlarged lymph nodes (short axis $\geq 10 \mathrm{~mm}$ ) on MRI were excluded. Patients underwent an SLN procedure with preoperative $99 \mathrm{~m}$ Tc-nanocolloid SPECT/CT-based SLN mapping. When fused datasets of the SPECT and MR images were created, SLNs could be identified on the MR image with accurate correlation to the histologic result of each individual SLN. An experienced radiologist, masked to histology, retrospectively reviewed all fused SPECT/MR images and scored morphologic SLN parameters on a standardized case report form. Logistic regression and receiver-operating curves were used to model the parameters against the SLN status. Results: In 75 cases, 136 SLNs were eligible for analysis, of which 13 (9.6\%) contained metastases (8 cases). Three parameters-short-axis diameter, long-axis diameter, and absence of sharp demarcation—significantly predicted metastatic invasion of nonenlarged SLNs, with quality-adjusted odds ratios of 1.42 (95\% confidence interval $[\mathrm{Cl}], 1.01-1.99), 1.28(95 \% \mathrm{Cl}, 1.03-1.57)$, and $7.55(95 \%$ $\mathrm{Cl}, 1.09-52.28)$, respectively. The area under the curve of the receiveroperating curves combining these parameters was $0.749(95 \% \mathrm{Cl}$, 0.569-0.930). Heterogeneous gadolinium enhancement, cortical thickness, round shape, or SLN size, compared with the nearest non-SLN, showed no association with metastases $(P=0.055-0.795)$. Conclusion: In cervical cancer patients without enlarged lymph nodes, selective evaluation of only the SLNs-for size and absence of sharp demarcationcan be used to noninvasively assess the presence of metastases.
\end{abstract}

Key Words: cervical cancer; sentinel lymph node; metastasis; SPECT/CT; MRI

J Nucl Med 2016; 57:551-556

DOI: 10.2967/jnumed.115.164780

\section{S} Institute as the first nodes to which malignant cells are likely to

\footnotetext{
Received Jul. 30, 2015; revision accepted Nov. 23, 2015.

For correspondence or reprints contact: Jacob P. Hoogendam, UMC Utrecht Cancer Center, F.05.126, Heidelberglaan 100, 3584 CX, Utrecht, The Netherlands.

E-mail: j.hoogendam@umcutrecht.nl

Published online Dec. 17, 2015.

COPYRIGHT (c) 2016 by the Society of Nuclear Medicine and Molecular Imaging, Inc.
}

spread from a primary tumor (1). There can be one SLN or more than one. Accurate assessment of SLNs in stage I/II cervical cancer patients is important because the presence or absence of metastases is a predictor for survival $(2,3)$. Also, on the basis of the SLN status, individualized decisions between radical hysterectomy or chemoradiation are possible, allowing for a potential morbidity reduction by avoiding double-modality treatment (4-6).

Before the SLN procedure, assessment of all lymph nodes can be attempted noninvasively by pelvic MRI. Current criteria, such as the commonly used 10-mm short-axis cutoff, are focused on achieving a high specificity, which is reported between $69 \%$ and $96 \%(7-11)$. Consequently, this focus limits sensitivity, which ranges from $27 \%$ to $71 \%$, indicating that normal-sized metastatic lymph nodes (i.e., false-negatives) are not uncommon $(12,13)$. This poses a clinical challenge because early-stage patients with nodes less than $10 \mathrm{~mm}$ usually do not undergo further noninvasive staging.

We hypothesized that by specifically focusing on SLNs, contrary to reviewing the entire pelvic lymphatic chain, the diagnostic accuracy for detecting metastases on MRI may be improved. This selective identification of SLNs on MRI can be achieved through fusion with the preoperatively created ${ }^{99 \mathrm{~m}} \mathrm{Tc}$ SPECT/CT-based SLN imaging, which visualizes the number and anatomic location of the bilateral SLNs (14).

In this study, we aimed to identify specific SLN parameters on pelvic MRI to explore the accuracy in preoperatively diagnosing SLN metastases in early-stage cervical cancer patients. We focused on patients with short-axis nodes $(<10 \mathrm{~mm})$ on MRI, because in many centers these are considered nonsuggestive and do not undergo further lymph node assessment with PET/CT before their intraoperative SLN procedure or lymphadenectomy $(15,16)$.

\section{MATERIALS AND METHODS}

\section{Research Population}

We reanalyzed preoperative MRI datasets in a retrospective cohort of consecutive stage IA1-IIB1 cervical cancer patients, treated surgically between March 2011 and February 2015 in our tertiary gynecologic oncology referral center. Subjects were eligible for inclusion when cervical cancer was histologically proven and preoperative pelvic MRI was performed and contained only lymph nodes with a short axis of less than $10 \mathrm{~mm}$. In addition, an intraoperative SLN procedure with preoperative SPECT/CT mapping had to have been completed. Subjects were excluded if no SLN was detected on SPECT/CT or if a concurrent second primary malignancy was diagnosed. The institutional review board approved this retrospective study, and the requirement to obtain informed consent was waived. This report follows the 
STARD guideline for diagnostic accuracy studies (supplemental data, available at http://jnm.snmjournals.org) (17).

\section{SLN Procedure}

Our SLN methodology has recently been published in detail (18). To summarize, at a standardized $17 \mathrm{~h}$ before surgery, 202-290 MBq of ${ }^{99 \mathrm{~m} T c-n a n o c o l l o i d}$ were submucosally injected into the 4 quadrants of the cervix directly surrounding the tumor. Ninety minutes after injection, a SPECT/CT scan was acquired on a single integrated system (Symbia T16; Siemens). A board-certified nuclear medicine physician reviewed the datasets and reported the number of SLNs and locations of the SLNs.

At surgery, the SLN procedure typically precedes a pelvic lymphadenectomy and radical hysterectomy or radical vaginal trachelectomy. On incision of the retroperitoneum, $0.5-1.0 \mathrm{~mL}$ of patent blue dye (Bleu Patenté; Guerbet Group) were injected intracervically directly surrounding the tumor with the same 4-quadrant technique as used before surgery, allowing for both visual and $\gamma$ probe-aided intraoperative SLN identification.

Histologic SLN assessment was performed according to an institutional protocol. After an optional frozen section analysis at the time of operation, definitive histology by paraffin embedding and serialstep sectioning at $150-\mu \mathrm{m}$ intervals was performed. Slides $(5 \mu \mathrm{m})$ were stained with hematoxylin and eosin, followed by routine immunohistochemical staining with cytokeratin AE1/AE3 antibodies. A pathologist who specializes in gynecologic oncology reviewed all specimens. Both micrometastases $(0.2-2 \mathrm{~mm})$ and macrometastases $(>2 \mathrm{~mm})$ indicated a tumor-positive SLN. In contrast, isolated tumor cells $(<0.2 \mathrm{~mm})$ were considered as tumor-negative, clinically as well as for the purpose of this study (3).

\section{MRI-Based SLN Review}

As part of routine noninvasive staging, a preoperative pelvic MRI was obtained on a 1.5-T wide-bore MRI system (Achieva/Ingenia; Phillips Health Care). To reduce bowel movements, $1.0 \mathrm{mg}$ of glucagon was administered intramuscularly. The standardized protocol consists of a T2-weighted turbo spin echo sequence in both the transversal (repetition time [TR], 6,687 ms; echo time [TE], $100 \mathrm{~ms}$; field of view [FoV], $250 \mathrm{~mm}$; matrix, $512 \times 512$; flip angle, $90^{\circ}$; slice thickness/gap, 4/0 mm) and the sagittal plane (TR, 2,800 ms; TE, $100 \mathrm{~ms}$; FoV, $250 \mathrm{~mm}$; matrix, $512 \times 512$; flip angle, $90^{\circ}$; slice thickness/gap, 4/0 mm); a transversal fat-saturated T1-weighted high-resolution isotropic volume excitation (THRIVE) sequence before and after intravenous gadolinium injection (for both: TR, 5.5 ms; TE, $2.7 \mathrm{~ms}$; FoV, $395 \mathrm{~mm}$; matrix, $512 \times 512$; flip angle, $10^{\circ}$; slice thickness/gap, 3/0 mm); a transversal proton density sequence (TR, 1,705 ms; TE, $20 \mathrm{~ms}$; FoV, $450 \mathrm{~mm}$; matrix, $512 \times 512$; flip angle, $90^{\circ}$; slice thickness/gap, 6/0.6 mm), which covers the complete abdomen; and a transversal diffusion-weighted sequence.

To identify the SLNs on MRI, an approach with fused datasets, generated in the dedicated fusion software platform syngo.via (version 1.1.0.16; Siemens), was adopted. First, a fused dataset was created from the CT and T1-/T2-weighted MRI datasets using the built-in automatic rigid alignment function. The accuracy of this fusion was visually assessed on the criterion of exact 3-dimensional superposition of the ossal anatomy. In rare instances, additional manual alignment was performed. This CT/MRI fusion, and the inherent anatomic link between SPECT and CT, enables a fusion matched for anatomy between the SPECT and T1-/T2-weighted MRI datasets (Fig. 1). Stills of SLNs on fused SPECT/MRI datasets were created and exported to PACS (SECTRA A.B.).

MRI datasets were prospectively reviewed for SLN morphology by a board-certified radiologist with $5 \mathrm{y}$ of experience in gynecologic oncology. Reviews were based on all MRI sequences available and
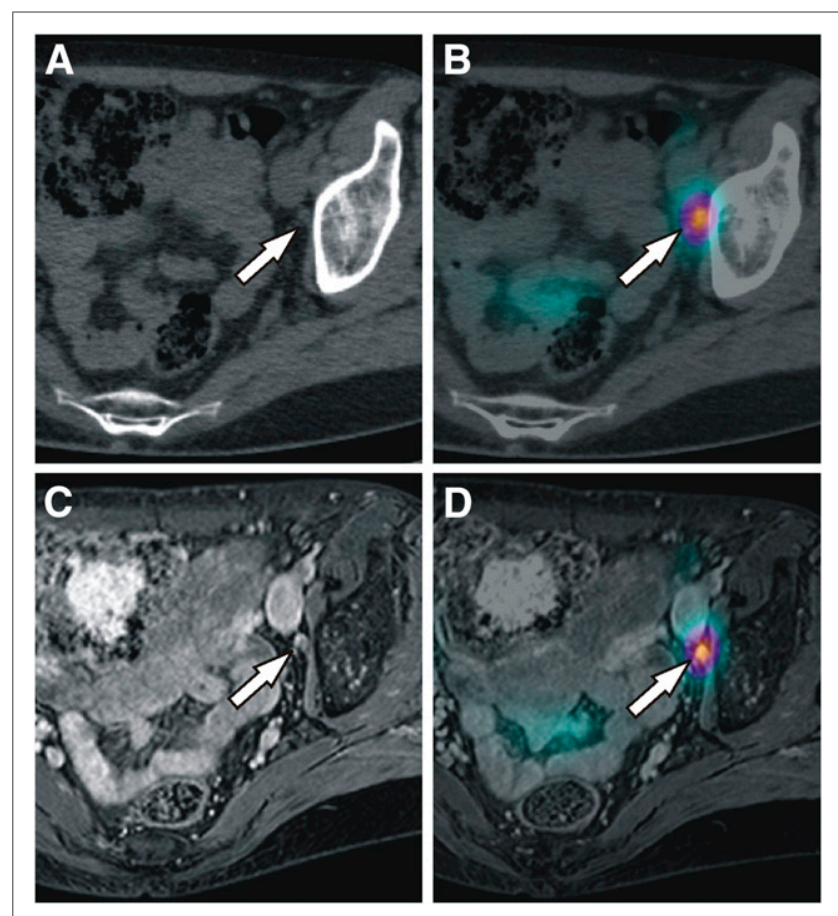

FIGURE 1. Illustration of fusion methodology in exemplary 53-y-old patient with stage IIA squamous cell carcinoma of the cervix. Preoperative SLN imaging with CT (A) as part of SPECT/CT (B) visualized SLN (arrow) in left obturator fossa. Gadolinium-enhanced T1-weighted MRI (C) performed during routine work-up can, via anatomy-guided fusion between CT and MRI, be used to create SPECT/MRI fused image (D). At histology, this SLN was found to be tumor-negative.

conducted at a clinical PACS workstation. The reviewing radiologist was masked to the histopathologic SLN status and all supporting clinical data, though aware of the study aim. A standardized case report form was used for scoring each SLN and included the following parameters: anatomic location; short- and long-axis diameter; shape; cortical thickness (when applicable); and presence or absence of sharp demarcation, uniform gadolinium enhancement, fatty center, or central necrosis. In addition, the short- and long-axis diameters of the nearest—in any direction-non-SLN was recorded. Image quality of the MRI dataset was rated on a 5-point Likert scale.

\section{Statistical Analysis}

Statistical calculations were performed with the Statistical Package for the Social Sciences (version 21.0.0; International Business Machines). Baseline characteristics were analyzed in a casewise approach.

For the per-node analysis, logistic regression was used to model the parameters scored on MRI against the histopathologically assessed SLN status (i.e., the reference standard). This produces uniformly comparable effect sizes in the form of odds ratios and significance testing with a single test (i.e., the Wald test), regardless of the data type (continuous or categoric). The scored MRI quality was added as an additional explanatory variable to the multivariate analysis to adjust the diagnostic odds ratios for the quality of the MRI dataset. The included variables had only $1.9 \%$ missing values, and no outliers were modified. Significant parameters, defined as a $P$ value of less than 0.050 , were combined in a single multivariable model. Predicted individual probabilities were incorporated into a receiver-operating curve (ROC) of which the area under the curve (AUC) was calculated, including $95 \%$ confidence intervals (95\% CIs). 


\section{Research Population}

Seventy-five patients were included, for whom the baseline characteristics are outlined in Table 1 . In 60 patients $(80.0 \%)$, bilateral detection (i.e., $\geq 1$ SLN per hemipelvis) was achieved on SPECT/CT. In total, 164 SLNs were visualized on SPECT/CT, of which 141 SLNs $(86.0 \%)$ could be unequivocally matched to a lymph node visible on MRI. The 23 excluded SLNs were all histologically negative for metastases and came from typical anatomic locations. An additional 5 SLNs (3.5\%) could not be intraoperatively identified and were also excluded from the analysis. Of the remaining 136 SLNs, 13 SLNs $(9.6 \%)$ in 8 of 75 patients $(10.7 \%)$ contained micro- or macrometastases. Only 1 tumor-positive SLN showed extranodal growth. Tumor-positive non-SLNs were found in 2 patients $(2.7 \%)$, both of whom had macrometastases in their ipsilateral SLN (i.e., no false-negative cases). Isolated tumor cells were identified in 6 SLNs (3.7\%) from 6 patients, of whom only 1 had a contralateral positive SLN. All 6 cases staged as IB2 or greater (e.g., patients underwent the SLN procedure to individualize the radiotherapy field) exhibited bilaterally detected tumor-negative SLNs.

Of the 136 SLNs analyzed, 74 SLNs were located in the obturator fossa $(54.4 \%), 43$ at the external iliac artery $(31.6 \%), 4$ dorsal of the internal iliac artery (2.9\%), and 15 at various other locations $(11.0 \%)$.

The mean interval between MRI and the SLN procedure was $22.8 \pm 12.8 \mathrm{~d}$, and no treatment was administered in between. The image quality of the MRI datasets was graded as poor, limited, intermediate, good, or excellent in $0.0 \%(0 / 75), 1.3 \%(1 / 75), 18.7 \%$ (14/75), $50.7 \%$ (38/75), and $29.3 \%$ (22/75), respectively.

\section{Diagnostic Accuracy}

The scored MRI parameters are descriptively outlined in Table 2, and an example case is shown in Figure 2. After adjusting for MRI dataset quality, only the short-axis SLN diameter $(P=0.042)$, the long-axis SLN diameter $(P=0.023)$, and the absence of sharp SLN demarcation $(P=0.040)$ were significantly associated with a tumorpositive SLN (Table 2). Interestingly, the SLN was significantly larger-in both short- $(P<0.001)$ and long-axis $(P<0.001)$ diameter - than the anatomically nearest non-SLN whether the SLN was tumor-positive or not. The SLN shape ( $P=0.250-0.795)$, cortex thickness $(P=0.282-0.738)$, and presence or absence of heterogeneous gadolinium enhancement $(P=0.107)$ or fatty hilum $(P=$ $0.637)$ all showed no significant positive or negative association with nodal metastases.

A subgroup analysis on macrometastases, by recoding micrometastatic SLNs as negative, did not identify additional significant parameters.

The ROCs of the short-axis SLN diameter, long-axis SLN diameter, and absence of sharp SLN demarcation had an AUC of 0.668 (95\% CI, 0.528-0.808), 0.655 (95\% CI, 0.482-0.829), and 0.574 (95\% CI, 0.381-0.768), respectively. ROC plots are provided in the supplemental data. A short-axis SLN diameter cutoff of $5.6 \mathrm{~mm}$ yielded the highest combined sensitivity $(53.8 \%)$ and specificity $(81.3 \%)$. For the long-axis SLN diameter, this cutoff was $10.5 \mathrm{~mm}$ for the sensitivity (38.5\%) and specificity (94.3\%).

The moderate increase to an AUC of 0.749 (95\% CI, 0.569-0.930) for the multivariable model — based on all 3 MRI parameters-when compared with the AUCs of the individual parameters, is likely attributable to the significant correlation (Pearson r: 0.669, $P<$ 0.001 ) between the short and long axis of the SLN (i.e., multicolinearity).
Baseline Characteristics of Research Population

\begin{tabular}{|c|c|c|}
\hline Characteristic & $n$ & $\%^{*}$ \\
\hline \multicolumn{3}{|l|}{ Prior cervical procedure } \\
\hline LLETZ & 38 & 50.7 \\
\hline Conization & 8 & 10.7 \\
\hline LLETZ and onization & 2 & 2.7 \\
\hline None & 27 & 36.0 \\
\hline \multicolumn{3}{|l|}{ Stage } \\
\hline IA1 or IA2 & 5 & 6.7 \\
\hline IB1 & 64 & 85.3 \\
\hline IB2, IIA1, IIA2, or IIB1 & 6 & 8.0 \\
\hline \multicolumn{3}{|l|}{ Tumor histology primary tumor } \\
\hline Squamous cell carcinoma & 59 & 78.7 \\
\hline Adenocarcinoma & 12 & 16.0 \\
\hline Other & 4 & 5.3 \\
\hline \multicolumn{3}{|c|}{ Tumor differentiation primary tumor } \\
\hline Grade 1 & 10 & 13.3 \\
\hline Grade 2 & 35 & 46.7 \\
\hline Grade 3 & 22 & 29.3 \\
\hline Not defined & 8 & 10.7 \\
\hline \multicolumn{3}{|l|}{ LVSI in primary tumor } \\
\hline Present & 33 & 44.0 \\
\hline Absent & 42 & 56.0 \\
\hline \multicolumn{3}{|l|}{ SLN procedure approach } \\
\hline Laparotomy & 1 & 1.3 \\
\hline Conventional laparoscopy & 16 & 21.3 \\
\hline Robot-assisted laparoscopy & 58 & 77.3 \\
\hline \multicolumn{3}{|c|}{ SLN histology, per patient $(n=75)^{\dagger}$} \\
\hline No tumor & 62 & 82.7 \\
\hline Isolated tumor cells & 5 & 6.7 \\
\hline Micrometastasis & 2 & 2.7 \\
\hline Macrometastasis & 6 & 8.0 \\
\hline \multicolumn{3}{|c|}{ SLN histology, per sentinel node $(n=136)$} \\
\hline No tumor & 117 & 86.0 \\
\hline Isolated tumor cells & 6 & 4.4 \\
\hline Micrometastasis & 5 & 3.7 \\
\hline Macrometastasis & 8 & 5.9 \\
\hline
\end{tabular}

${ }^{*}$ Percentages are based on 75 cases unless otherwise noted.

${ }^{\dagger}$ Categorization is based on severest metastatic SLN load present within each patient.

LLETZ = large loop excision of the transformation zone; LVSI = lymphovascular space invasion.

Median age, with range in parentheses, was 39.5 y (25.3-81.2 y); median body mass index, with range in parentheses, was $23.5 \mathrm{~kg} / \mathrm{m}^{2}$ (18.3-41.0 kg/m²); and median follow-up, with range in parentheses, was $17 \mathrm{mo}(0-47 \mathrm{mo})$.

\section{DISCUSSION}

We have used SPECT/MRI fusion to enable a selective assessment of SLNs in early-stage cervical cancer patients. We purposely focused our analysis on nonenlarged, lymph nodes less than $10 \mathrm{~mm}$ 
TABLE 2

Results of Reviewed MRI Parameters

\begin{tabular}{|c|c|c|c|c|c|}
\hline \multirow[b]{2}{*}{ Parameter } & \multicolumn{2}{|c|}{ Measured result } & \multicolumn{3}{|c|}{ Diagnostic odds ratio for metastasis detection ${ }^{\star}$} \\
\hline & $\begin{array}{c}123 \text { tumor-negative } \\
\text { SLNs }\end{array}$ & 13 tumor-positive SLNs & Odds ratio & $95 \% \mathrm{Cl}$ & $P$ \\
\hline \multicolumn{6}{|l|}{ Median SLN size (mm) } \\
\hline Short-axis diameter & 4.0 (range, 1.0-8.9) & 5.6 (range, 2.0-6.7) & 1.42 & $1.01-1.99$ & 0.042 \\
\hline Long-axis diameter & 7.0 (range, $1.0-14.3$ ) & 8.2 (range, 3.7-16.0) & 1.28 & $1.03-1.57$ & 0.023 \\
\hline Ratio long relative to short axis ${ }^{\dagger}$ & 1.7 (range, 1.0-4.6) & 1.9 (range, 1.0-3.0) & 0.75 & $0.32-1.73$ & 0.497 \\
\hline \multicolumn{6}{|l|}{ SLN (\%) categorized by shape ${ }^{\ddagger}$} \\
\hline Bean & $47(38.2 \%)$ & $3(23.1 \%)$ & 0.45 & $0.12-1.75$ & 0.250 \\
\hline Disk & $33(26.8 \%)$ & $6(46.2 \%)$ & 1.98 & $0.60-6.50$ & 0.262 \\
\hline Spheric/ball & $38(30.9 \%)$ & $3(23.1 \%)$ & 0.83 & $0.21-3.30$ & 0.795 \\
\hline Lobulated & $5(4.1 \%)$ & $1(7.7 \%)$ & 2.60 & $0.27-25.46$ & 0.412 \\
\hline \multicolumn{6}{|l|}{ SLN (\%) with $\ddagger$} \\
\hline Absence of sharp demarcation & $4(3.3 \%)$ & $2(15.4 \%)$ & 7.55 & $1.09-52.28$ & 0.040 \\
\hline $\begin{array}{l}\text { Heterogeneous gadolinium } \\
\text { enhancement }\end{array}$ & $17(13.8 \%)$ & $4(30.8 \%)$ & 3.06 & $0.79-11.89$ & 0.107 \\
\hline Fat hilum visible & $17(13.8 \%)$ & $1(7.7 \%)$ & 0.60 & $0.07-5.06$ & 0.637 \\
\hline Central necrosis & $0(0.0 \%)$ & $0(0.0 \%)$ & Not applicable & Not applicable & Not applicable \\
\hline Cystic component & $0(0.0 \%)$ & $0(0.0 \%)$ & Not applicable & Not applicable & Not applicable \\
\hline \multicolumn{6}{|l|}{ SLN (\%) categorized by cortex $\ddagger$} \\
\hline Cortex cannot be discerned & $90(73.2 \%)$ & $9(69.2 \%)$ & 0.59 & $1.56-2.24$ & 0.438 \\
\hline Cortex $<3 \mathrm{~mm}$ & $29(23.6 \%)$ & $3(23.1 \%)$ & 1.27 & $0.31-5.29$ & 0.738 \\
\hline Cortex $\geq 3 \mathrm{~mm}$ & $4(3.3 \%)$ & $1(7.7 \%)$ & 3.79 & $0.33-43.09$ & 0.282 \\
\hline \multicolumn{6}{|l|}{ Median nearest non-SLN size (mm) } \\
\hline Short-axis diameter & 3.0 (range, 1.0-9.0) & 3.5 (range, 1.0-5.2) & 1.35 & $0.95-1.92$ & 0.092 \\
\hline Long-axis diameter & 4.9 (range, 1.0-15.0) & 7.9 (range, 3.5-12.0) & 1.19 & $1.00-1.42$ & 0.055 \\
\hline $\begin{array}{l}\text { Ratio short axis relative to } \\
\text { short-axis SLN }\end{array}$ & 1.4 (range, 0.4-5.5) & 1.3 (range, 0.6-6.0) & 1.09 & $0.63-1.88$ & 0.755 \\
\hline $\begin{array}{l}\text { Ratio long axis relative to long- } \\
\text { axis } \mathrm{SLN}^{\dagger}\end{array}$ & 1.2 (range, 0.1-9.7) & 1.1 (range, 0.8-2.1) & 0.58 & $0.25-1.37$ & 0.216 \\
\hline $\begin{array}{l}{ }^{*} \text { Values are multivariable adjusted } \\
{ }^{\dagger} \text { Calculated variable based on scor } \\
{ }^{\ddagger} \text { Depending on either tumor-negati } \\
\text { SLNs, respectively. } \\
\text { LN = lymph node. }\end{array}$ & $\begin{array}{l}\text { for quality of MRI datas } \\
\text { red parameters. } \\
\text { ve or tumor-positive SL }\end{array}$ & $\begin{array}{l}\text { sets. } \\
\text { N status, percentages in } r\end{array}$ & measured result & columns are frac & tions of 123 or 13 \\
\hline
\end{tabular}

because in clinical practice these nodes are commonly considered nonmetastatic. Women with early-stage cervical cancer and nonenlarged nodes generally do not undergo PET/CT, unless there is an alternative indication (e.g., MRI findings) (15). This is in contrast to the routine use of PET/CT for advanced (stage III-IV) disease. Our approach was based on the premise that a focused review of a few lymph nodes - with the knowledge that these are the SLNs-instead of indiscriminately assessing all nonenlarged lymph nodes, could improve the detection of metastases.

We have shown that selective SLN assessment on MRI is feasible. The analysis further identified 3 clinically usable MRI parameters - the short- and long-axis diameters and the absence of a sharp demarcation-that all had a significant association with nodal metastases from cervical cancer. As expected in nodes less than $10 \mathrm{~mm}$, the univariable and combined diagnostic accuracy was only moderate at best, but reducing false-negatives in these nodes is an attractive clinical goal. Even after this short-axis selection of less than $10 \mathrm{~mm}$, SLN size remained significantly correlated with the odds of metastases. This is in line with Choi et al. $(n=55)$ who found a comparable result in a node-by-node analysis for the short axis of metastatic non-SLNs on MRI (19). Unfortunately, other studies often present only a single size cutoff, instead of maintaining it as a continuous variable (such as in a ROC analysis), which better reflects the inverse dependency between sensitivity and specificity.

The cervical cancer study by Choi et al. also observed significantly less often smooth margins in metastatic lymph nodes, regardless of their size (19). Likewise, absence of smooth margins has been linked by Brown et al. to nodal metastases in rectal cancer, with a sensitivity of $75 \%$ and specificity of $98 \%$. However, 

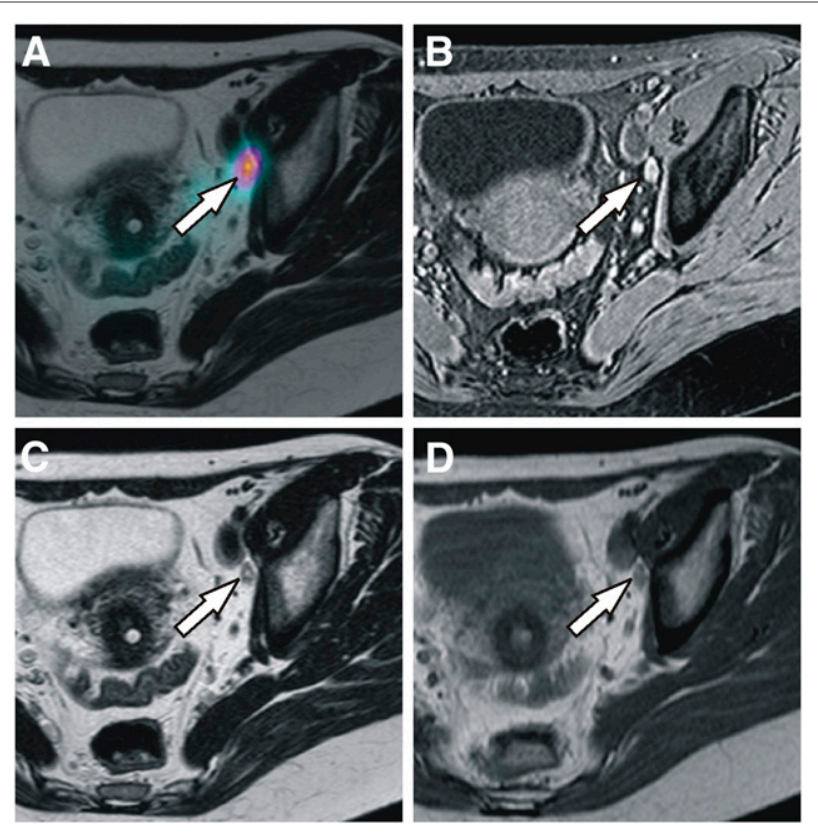

FIGURE 2. In a 39-y-old patient with stage IB1 adenosquamous cervical cancer, a SLN was found on fused SPECT/T2-weighted MRI (A) in left obturator fossa (arrow), in addition to 2 SLNs in right hemipelvis (not depicted). Left SLN, indeed radioactive and located in obturator fossa at surgery, showed 1.0-mm micrometastasis without extranodal growth at histology. SLN parameters were reviewed masked on T1-weighted (B), T2-weighted (C), and proton density sequence (D). SLN measured $6.0 \times 12.0 \mathrm{~mm}$ and was scored as having lobulated shape with absence of sharp demarcation of its margins and heterogeneous gadolinium enhancement pattern.

their inclusion of large metastatic lymph nodes - up to $15 \mathrm{~mm}-$ could have potentially influenced the margin assessment and inflated the diagnostic accuracy outcome; this is in contrast to the selective inclusion of small lymph nodes less than $10 \mathrm{~mm}$ in the current study (20). In terms of SLN shape, various authors consider spheric (i.e., round in a single orthogonal plane) lymph nodes intrinsically more suspicious than those with a bean or oval appearance $(13,21,22)$. In our results, both direct SLN shape judgments and long-/short-axis ratios did not have a significant association with metastatic invasion.

Although the intraoperative SLN procedure is invasive and laborious and carries a risk of adverse events, it remains diagnostically superior to conventional MRI assessment wherein all pelvic lymph nodes — not specifically SLNs—are reviewed. A meta-analysis from 2008 (72 studies, $n=5,042$ ) by Selman et al. reported a pooled sensitivity of $91.4 \%, 74.7 \%$, and $55.5 \%$ and a specificity of $100.0 \%$, $97.6 \%$, and $93.2 \%$ in detecting lymph node metastases from cervical cancer for the SLN procedure, PET/CT, and MRI, respectively (23). Studies on MRI published after this meta-analysis have shown comparable results for its diagnostic accuracy in cervical cancer $(7,24-$ 28 ). However, a recent study on the intraoperative SLN procedure by Cibula et al. ( 8 centers, $n=645$ ) reported a further increase to $97 \%$ sensitivity when SLNs were bilaterally detected and histopathologically ultrastaged (2).

Certain limitations of our study merit further explanation. First, the number of tumor-positive SLNs (i.e., events) was limited to 13 SLNs in 8 cases. Although this represents the normal incidence of metastases in a stage I/II cohort conditioned on short-axis lymph

nodes less than $10 \mathrm{~mm}$, the statistical power to prove associations is restricted. Second, we adopted a single reviewer approach, which inherently challenges reproducibility when compared with consensus statements by multiple independent reviewers. However, this issue is at least partly alleviated by adequate blinding, the use of measurable criteria (e.g., SLN diameter), and a review by an experienced radiologist specialized in gynecologic and oncologic imaging. Third, our approach still requires CT for accurate anatomy-based fusion of the SPECT dataset with the work-up MRI. However, this can be overcome when hybrid SPECT/MRI scanners become commercially available in the future. Finally, our research is exploratory and requires external validation in larger series.

\section{CONCLUSION}

We have shown that a SPECT/MRI review specifically focused on detecting cervical cancer metastases in short-axis SLNs less than $10 \mathrm{~mm}$ is feasible and-as hypothesized-may reduce the number of false-negatives in these patients. Although it cannot match the intraoperative SLN procedure in terms of accuracy, a clinical benefit is that it may allow for the selection of early-stage patients for PET/CT. Also, our MRI-based SLN assessment noninvasively provides the radiologist with information on the nodal site at which metastatic disease may first appear, deemphasizing non-SLNs in the review process. Three easy-to-use SLN parameters were identified, namely the short- and long-axis diameter and the absence of sharp demarcation, which could be of potential value for future research or patients wherein SLNs cannot be intraoperatively retrieved. These exploratory results justify larger and prospective series focused on the value of selective SLN assessment on MRI.

\section{DISCLOSURE}

The costs of publication of this article were defrayed in part by the payment of page charges. Therefore, and solely to indicate this fact, this article is hereby marked "advertisement" in accordance with 18 USC section 1734 . No potential conflict of interest relevant to this article was reported.

\section{REFERENCES}

1. Sentinel lymph node biopsy. National Cancer Institute website. http://www.cancer. gov/about-cancer/diagnosis-staging/staging/sentinel-node-biopsy-fact-sheet\#q2. Updated August 11, 2011. Accessed January 21, 2016.

2. Cibula D, Abu-Rustum NR, Dusek L, et al. Bilateral ultrastaging of sentinel lymph node in cervical cancer: lowering the false-negative rate and improving the detection of micrometastasis. Gynecol Oncol. 2012;127:462-466.

3. Cibula D, Abu-Rustum NR, Dusek L, et al. Prognostic significance of low volume sentinel lymph node disease in early-stage cervical cancer. Gynecol Oncol. 2012;124:496-501.

4. Baalbergen A, Veenstra Y, Stalpers L. Primary surgery versus primary radiotherapy with or without chemotherapy for early adenocarcinoma of the uterine cervix. Cochrane Database Syst Rev. 2013;1:CD006248.

5. Landoni F, Maneo A, Colombo A, et al. Randomised study of radical surgery versus radiotherapy for stage Ib-IIa cervical cancer. Lancet. 1997;350:535-540.

6. Eiriksson LR, Covens A. Sentinel lymph node mapping in cervical cancer: the future? BJOG. 2012;119:129-133.

7. Chung HH, Kang KW, Cho JY, et al. Role of magnetic resonance imaging and positron emission tomography/computed tomography in preoperative lymph node detection of uterine cervical cancer. Am J Obstet Gynecol. 2010;203:156.e1-156.e5.

8. Hori M, Kim T, Murakami T, et al. Uterine cervical carcinoma: preoperative staging with 3.0-T MR imaging-comparison with 1.5-T MR imaging. Radiology. 2009;251:96-104.

9. Testa AC, Ludovisi M, Manfredi R, et al. Transvaginal ultrasonography and magnetic resonance imaging for assessment of presence, size and extent of invasive cervical cancer. Ultrasound Obstet Gynecol. 2009;34:335-344. 
10. Sheu M, Chang C, Wang J, Yen M. MR staging of clinical stage I and IIa cervical carcinoma: a reappraisal of efficacy and pitfalls. Eur J Radiol. 2001;38:225-231.

11. Choi HJ, Roh JW, Seo SS, et al. Comparison of the accuracy of magnetic resonance imaging and positron emission tomography/computed tomography in the presurgical detection of lymph node metastases in patients with uterine cervical carcinoma: a prospective study. Cancer. 2006;106:914-922.

12. Sala E, Rockall AG, Freeman SJ, Mitchell DG, Reinhold C. The added role of MR imaging in treatment stratification of patients with gynecologic malignancies: what the radiologist needs to know. Radiology. 2013;266:717-740.

13. Lai G, Rockall AG. Lymph node imaging in gynecologic malignancy. Semin Ultrasound CT MR. 2010;31:363-376.

14. Hoogendam JP, Veldhuis WB, Hobbelink MG, Verheijen RH, van den Bosch MA, Zweemer RP. Technetium-99m SPECT-CT versus planar lymphoscintigraphy for preoperative sentinel lymph node detection in cervical cancer: a systematic review and meta-analysis. J Nucl Med. 2015;56:675-680.

15. Chou HH, Chang TC, Yen TC, et al. Low value of $\left[{ }^{18} \mathrm{~F}\right]$-fluoro-2-deoxy-Dglucose positron emission tomography in primary staging of early-stage cervical cancer before radical hysterectomy. J Clin Oncol. 2006;24:123-128.

16. Driscoll DO, Halpenny D, Johnston C, Sheehy N, Keogan M. ${ }^{18}$ F-FDG-PET/CT is of limited value in primary staging of early stage cervical cancer. Abdom Imaging. 2015;40:127-133.

17. Bossuyt PM, Reitsma JB, Bruns DE, et al. Standards for reporting of diagnostic accuracy: towards complete and accurate reporting of studies of diagnostic accuracy: the STARD initiative. BMJ. 2003;326:41-44.

18. Hoogendam JP, Hobbelink MG, Veldhuis WB, Verheijen RH, van Diest PJ, Zweemer RP. Preoperative sentinel node mapping with ${ }^{99 \mathrm{~m}} \mathrm{Tc}-$-nanocolloid SPECT-CT significantly reduces the intraoperative sentinel node retrieval time in robot assisted laparoscopic cervical cancer surgery. Gynecol Oncol. 2013;129: 389-394.
19. Choi HJ, Kim SH, Seo SS, et al. MRI for pretreatment lymph node staging in uterine cervical cancer. AJR. 2006;187:W538-W543.

20. Brown G, Richards CJ, Bourne MW, et al. Morphologic predictors of lymph node status in rectal cancer with use of high-spatial-resolution MR imaging with histopathologic comparison. Radiology. 2003;227:371-377.

21. Kim SH, Kim SC, Choi BI, Han MC. Uterine cervical carcinoma: evaluation of pelvic lymph node metastasis with MR imaging. Radiology. 1994;190:807-811.

22. Torabi M, Aquino SL, Harisinghani MG. Current concepts in lymph node imaging. J Nucl Med. 2004;45:1509-1518.

23. Selman TJ, Mann C, Zamora J, Appleyard TL, Khan K. Diagnostic accuracy of tests for lymph node status in primary cervical cancer: a systematic review and meta-analysis. CMAJ. 2008;178:855-862.

24. Zhang W, Zhang J, Yang J, et al. The role of magnetic resonance imaging in pretreatment evaluation of early-stage cervical cancer. Int $J$ Gynecol Cancer. 2014;24:1292-1298.

25. Lv K, Guo HM, Lu YJ, Wu ZX, Zhang K, Han JK. Role of ${ }^{18}$ F-FDG PET/CT in detecting pelvic lymph-node metastases in patients with early-stage uterine cervical cancer: comparison with MRI findings. Nucl Med Commun. 2014;35:12041211.

26. Klerkx WM, Veldhuis WB, Spijkerboer AM, et al. The value of 3.0 tesla diffusionweighted MRI for pelvic nodal staging in patients with early stage cervical cancer. Eur J Cancer. 2012;48:3414-3421.

27. Hong KS, Ju W, Choi HJ, Kim JK, Kim MH, Cho KS. Differential diagnostic performance of magnetic resonance imaging in the detection of lymph node metastases according to the tumor size in early-stage cervical cancer patients. Int J Gynecol Cancer. 2010;20:841-846.

28. Mitchell DG, Snyder B, Coakley F, et al. Early invasive cervical cancer: MRI and CT predictors of lymphatic metastases in the ACRIN 6651/GOG 183 intergroup study. Gynecol Oncol. 2009;112:95-103. 